\title{
DESIGN DE EXPERIÊNCIA DE APRENDIZAGEM: AVALIAÇÃO DO MODELO ADDIE E CONTRIBUIÇÕES PARA O ENSINO A DISTÂNCIA
}

http://dx.doi.org/10.5902/2318133831922

\author{
Andrea Moura da Costa Souza ${ }^{1}$ \\ Maria Lucijane Gomes de Oliveira² \\ Marcos Antônio Martins Lima ${ }^{3}$ \\ Blússia Tétis Brito Batista ${ }^{4}$
}

Resumo

Refletir sobre o design da aprendizagem nos remete a entender como o discente apreende para que os processos de ensino e aprendizagem ocorram de modo eficaz. O papel do professor se mostra importante em todo o processo, pois este irá proporcionar ao discente uma experiência orientada para que o aprendizado seja alcançado. O objetivo desta pesquisa é descrever o modelo Addie - análise, design, desenvolvimento, implementação e avaliação - e avaliar as suas contribuições para prática docente, fazendo uso do design de experiência de aprendizagem no ensino a distância. Conclui-se que o modelo Addie se apresenta como importante ferramenta no planejamento pedagógico, por meio do qual o professor precisa cumprir as etapas para então avaliar a aprendizagem do estudante.

Palavras-chave: design de experiência; avaliação; modelo Addie.

\section{LEARNING EXPERIENCE DESIGN: EVALUATION OF THE ADDIE MODEL AND CONTRIBUTIONS FOR DISTANCE EDUCATION}

\section{Abstract}

Reflecting on the design of learning, leads us to understand how the learner grasps for this learning process to occur effectively. The role of the teacher is important in the whole process, because it will provide the student with a learning experience that is achieved. The purpose of this research is to describe the Addie model - Analysis, design, development, implementation and evaluation - and evaluate their contributions to teaching practice, making use of the design of learning experience in distance learning higher education. We conclude that the Addie model is an important tool in pedagogical planning. In which, the teacher needs to complete the steps all steps to then evaluate the student learning.

Key-words: experience design; evaluation; model Addie.

\footnotetext{
1 Universidade Federal do Ceará, Brasil. E-mail: andrea.souza@ifce.edu.br.

2 Universidade Federal do Ceará, Brasil. E-mail luciijanne.oliveira@gmail.com

3 Universidade Federal do Ceará, Brasil. E-mail: marcos.a.lima@terra.com.br.

${ }^{4}$ Universidade Federal do Ceará, Brasil. E-mail: blussia@hotmail.com.
} 


\section{Introdução}

termo design está cada vez mais presente no âmbito educacional. É possível identificar o uso do termo no ensino, na instrução, no treinamento e na aprendizagem. Pensar no design da aprendizagem, atualmente, se apresenta propício à constituição de uma estratégia que está centrada no discente, em que o docente precisa entender a forma de aprendizagem e proporcionar ao discente uma experiência orientada e capaz de alcançar o objetivo proposto, ou seja, o aprendizado. Esse artigo tem o intuito de descrever o modelo Addie e avaliar seu uso no design de experiência de aprendizagem.

$\mathrm{Na}$ formação e na concepção de módulos e-learning, o modelo Addie é muito requisitado. Este acrônimo é formado pela primeira letra de cada fase do método: análise; design; desenvolvimento; implementação e avaliação. Cada etapa é um pré-requisito para a próxima etapa e tem um papel importante para que o objetivo do projeto pedagógico seja alcançado com sucesso.

Ao se considerar as quatro importantes teorias pedagógicas, segundo Libâneo (1990) e Bonfim (1998) percebe-se que na pedagogia tradicional prevaleceu de forma inquestionável entre os anos 1889-1930. Na perspectiva dessa pedagogia o processo de ensino e aprendizagem acontece com o acúmulo de informações proveniente dos conteúdos dados pelos docentes, sendo assim, esse período o aprender ficou caracterizado como um exercício de memorização.

Em 1930 surgiu a pedagogia nova no Brasil. Com ela se descartou os fundamentos que enquadram o pensamento tradicional da pedagogia que se baseou no acúmulo do conhecimento e na forma de disseminação do ensino e aprendizado que não considerava as particularidades de cada indivíduo. A pedagogia nova foi a forma encontrada para entender o indivíduo no seu processo de desenvolvimento e maneira igualitária entre os indivíduos.

Nos anos 1960 e 1970 surgiu, no Brasil, a pedagogia tecnicista. Essa escola pedagógica surgiu nos Estados Unidos e refletiu o contexto econômico e pragmático da época. Quando surgiu no Brasil o tecnicismo cumpriu o mesmo propósito, ou seja, buscou atender aos interesses da produção capitalista e da racionalidade do trabalho que vivenciava o país, tornando o processo educativo operacional e voltado para o trabalho e para indústria.

A pedagogia libertadora, na década de 1960, apareceu como resultado do fortalecimento da classe proletária no Brasil e o êxodo dos agricultores das zonas rurais. Tendo como um dos precursores dessa pedagogia Paulo Freire, essa pedagogia buscou responsabilizar o discente pelo seu aprendizado, assim como formar um cidadão mais consciente de suas necessidade e realidade.

$\mathrm{O}$ design de aprendizagem envolve as atividades de aprendizagem e para que a modelagem alcance seu resultado, os cenários de aprendizagem são previstos visando a necessidade do discente. Entender a forma que os discentes aprendem facilitará a construção do curso ou disciplina. Desse modo, tornar-se necessário avaliar a gestão do design de atividades de aprendizagem levando em consideração os aspectos do modelo Addie - Análise, design, desenvolvimento, implementação e avaliação - e suas contribuições para prática docente no ensino superior. 
A metodologia empregada constitui-se como bibliográfica e está centrada nos seguintes autores: Neto e Hesketh (2009), Luckesi (2011), Filatro (2008), Lima (2008), Gomes e Silva (2016).

\section{Avaliação e design de experiências de aprendizagem}

O design de experiências de aprendizagem fundamenta-se na exploração da fonte criativa, utilizando ferramentas do design para que se obtenha soluções de diversos tipos de problemas.

Tratando o ser humano como centro do processo, o design de experiências e aprendizagem é utilizado para que os alunos consigam compreender, absorver e desenvolver uma nova forma de pensar. Busca adaptar a aprendizagem conforme características e expectativas de cada indivíduo. Dessa forma, visa a conhecer suas necessidades, desejos, esperanças e todo o contexto social em que está inserido.

Conceitualmente, design, é a arte de solucionar problemas de forma criativa. (Gomes e Silva, 2016). Para entender o uso do design na aprendizagem precisamos avaliar e para isso precisamos entender o conceito desse termo.

O termo avaliar, etimologicamente, origina-se do latim avaliare, que significa mensurar, dar valor, ou seja, compreender o processo de alcance de competências e habilidades relacionadas ao conhecimento com o intento de melhoria contínua do processo educativo. De acordo com Tyler (1974), a avaliação deve incidir no desenvolvimento permanente e coletivo, ocasionando melhorias gradativas nas práticas desenvolvidas no ambiente de aprendizagem.

Segundo Lima (2008),

o conceito de avaliar está referenciado no processo de cognição do ser humano, ou seja, uma função psicológica própria humana. A avaliação representa uma posição, um julgamento sobre a realidade vivenciada com o intuito de representar uma nova tomada de decisão acerca do objeto estudado. Assim, delimita a avaliação como o engendramento de um novo devir para nossa existência, dotado de convicção de verdade universalmente reconhecida. (p. 200)

Desse modo, a avaliação da aprendizagem possibilita a tomada de decisão e a melhoria da qualidade de ensino, orientando as práticas de ensino e a necessidade de regulações constantes (Kraemer, 2009; Luckesi, 2011). Nesse sentido, Caldeira (2000) afirma que

a avaliação é um meio e não um fim em si mesma; está delimitada por uma determinada teoria e por uma determinada prática pedagógica. Ela não ocorre num vazio conceitual, mas está dimensionada por um modelo teórico de sociedade, de homem, de educação e, consequentemente, de ensino e de aprendizagem, expresso na teoria e na prática pedagógica. ( $p$. 122)

Assim sendo, a avaliação é parte integrante do processo ensino e aprendizagem exigindo dos profissionais envolvidos conhecimento e capacidade de observação. Devese, portanto, considerar "a singularidade das situações e os acontecimentos como únicos. Gage (1979) sugere uma estrutura com dimensões ligadas a eficácia do ensino com 
diferentes matérias que vão do geral ao particular e permite considerar as especificidades do processo educativo" (Souza, 2016, p. 39). Contudo, a avaliação é um auxílio para estabelecer objetivos e metas, desenvolvimento dos alunos, a efetividade do processo e as possíveis mudanças para se realinhar com a finalidade de garantir sua efetividade.

Segundo Neto e Hesketh (2009) o design de atividade de aprendizagem "focaliza a criação, a elaboração e a oferta de uma série de ações que os alunos realizam durante sua rota de aprendizagem". Essa metodologia pretende "sistematizar uma proposta educacional centrada na ação de alunos e professores, deslocando o foco da mera transmissão de conteúdos para as atividades realizadas efetivamente pelos agentes de aprendizagem" (Neto e Hesketh, 2009, p. 97).

De acordo com Gomes e Silva (2016) "o planejamento adequado é fundamental para dar conta de todos os detalhes e permitir a execução de práticas de ensino" (p. 80). Assim, tendo em vista a necessidade de um planejamento mais detalhado, com abordagens metodológicas e técnicas bem definidas, que levem em consideração análise do contexto, levantamento das restrições, caracterização do público-alvo e condições do processo de ensino-aprendizagem, o modelo Addie surgiu como uma estratégia viável de auxílio ao planejamento da ação educacional.

\section{O planejamento pedagógico e o modelo Addie}

O planejamento do trabalho pedagógico tornou-se ainda mais complexo desde avanço das tecnologias digitais no ambiente educacional, contribuindo para novos discursos e novos paradigmas. O perfil dos usuários aprendizes também tem se modificando: estão menos motivados a copiar, ouvir, prestar atenção de forma estática e mais motivados a criar, projetar, construir, e desse modo, aprender de forma colaborativa.

Assim sendo, o planejamento pedagógico, segundo Gomes e Silva (2016),

pode ser útil não apenas para o uso de tecnologias na concepção de novas formas de aprendizagem. Ela pode ser também uma forma radical de conceber a experiência de aprendizagem envolvendo cenários, espaços, artefatos e processos avaliativos focados em experiências criativas e inovadoras. $O$ design pode contribuir muito para criarmos as melhores condições para que a aprendizagem ocorra de forma mais efetiva no interior das instituições de ensino e impactem positivamente a sociedade. (p. 145)

Nesse contexto, os autores apresentam o raciocínio do design como um novo procedimento para criação de ambientes de aprendizagem a partir de experiências criativas, ou seja, novas formas de ensino-aprendizagem fundamentadas em experiências significativas.

Raabe et al. (2016) enfatiza que

no campo pedagógico estamos em um período de valorização das vertentes educacionais que incentivam o estudante a se tornar protagonista de sua aprendizagem. Criar, construir, conceber, desenvolver, montar, combinar, projetar, testar, avaliar, revisar, vêm gradativamente se tornando verbos utilizados nos objetivos de aprendizagem dos planos de 
aula dos docentes e que elucidam que tipo de estudante deseja-se auxiliar a desenvolver. Multiplicam-se nas escolas as iniciativas que usam a abordagem baseadas em projetos, aprendizagem baseada em problemas e práticas alinhadas ao design. (p. 12)

Desse modo, o trabalho pedagógico consiste desde elaboração do planejamento didático, da seleção de técnicas e estratégias de metodologias de ensino, da seleção de conteúdos didáticos, considerando as características do usuário-aprendiz, e gera reflexões e embates relevantes que colaboram com o processo de aprendizagem

De acordo com Filatro (2008) as estratégias utilizadas no planejamento dos materiais didáticos utilizam modelos de produção e desenvolvimento de acordo com o contexto o qual se aplicam. De fato, os modelos perpassam desde concepção, planejamento e gestão das unidades temáticas.

O modelo Addie apresenta-se como um plano seguro para criação de recursos educacionais para módulos de ensino a distância. Neste modelo, as ações educacionais consistem no processo de cinco fases, a saber: análise, design, desenvolvimento, implementação e avaliação (Filatro, 2008). Diferente do modelo proposto por Stufflebeam (2003) que propôs somente quatro fases, seu modelo de avaliação foi denominado Cipp context, input, process, product - e como percebemos é uma adaptação da teoria geral dos sistemas do biólogo Bertalanffy (2008) que remete a correlação entre partes.

$\mathrm{Na}$ figura 1 temos o modelo Addie, que foi adotado por muitos autores, como Lebrun (2007) e apresenta de forma racional e sequencial um modelo de avaliação que permite de forma sólida resolver problemas voltados a aprendizagem.

Figura 2

Modelo Addie.

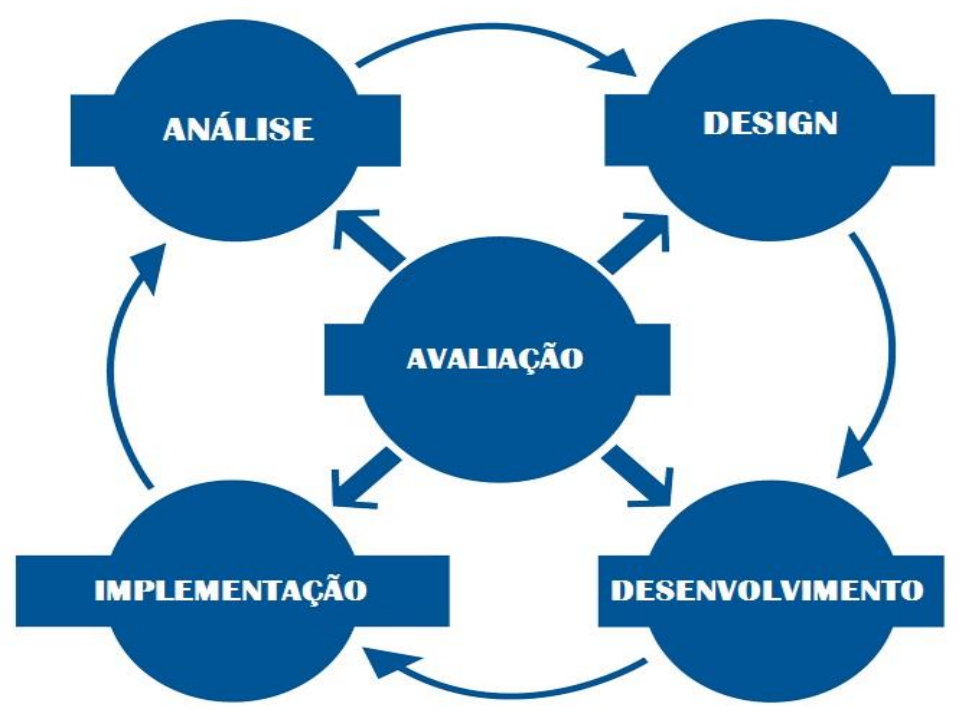

Fonte: adaptado de Filatro (2008).

A fase inicial, Análise, consiste em "entender o problema educacional e projetar uma solução aproximada" (Filatro, 2008, p. 28) ou seja, permite identificar as necessidades da ação educacional para que uma determinada situação didática seja realizada. Nesse 
momento faz-se uma análise contextual, mapeando algumas restrições e possibilidades para o processo de aprendizagem, considerando o perfil do público-alvo e selecionando as estratégias e metodologias que serão utilizadas no decorrer da ação educacional.

A fase seguinte, Design, compreende o "planejamento e o design da situação didática propriamente dita, com o mapeamento e sequenciamento dos conteúdos a serem trabalhados" (Filatro, 2008, p. 28). Nesta etapa define-se as estratégias de aprendizagem para 0 alcance dos objetivos pretendidos, inclui a seleção de mídias e recursos audiovisuais a fim de tornar o ambiente escolar mais atraente e interativo.

A fase de desenvolvimento compreende "a produção e a adaptação de recursos e materiais didáticos impressos e/ou digitais, a parametrização de ambientes virtuais e a preparação de suportes pedagógicos, tecnológicos e administrativos" (Filatro, 2008, p. 30). A fase posterior, Implementação, constitui a "situação didática propriamente dita" (Filatro, 2008, p. 30). É o momento em que se coloca em prática toda ação planejada.

Por último, a fase de Avaliação, que inclui as considerações sobre a efetividade da solução proposta, bem como revisão das estratégias implementadas. De acordo com Gomes e Silva (2016)

a avaliação da efetividade deve permitir identificar indícios de ganhos obtidos com o esforço para planejar e executar uma experiência de aprendizagem. [...] esses ganhos podem ser a aprendizagem de conceitos ou o desenvolvimento de habilidades e competências. Esses indícios podem ser medidos de forma continuada ou ao final de um ciclo. (p. 128)

Nela avalia-se tanto a solução educacional, quanto os resultados de aprendizagem dos alunos" (Filatro, 2008, p. 31). Esta fase inclui informações sobre a efetividade de experiências de aprendizagem. Como também o processo como um todo, onde se avalia "durante as etapas e entre as etapas e no fim do processo de implementação" (Lebrun, 2007, p. 89.)

\section{Contribuições do modelo Addie e design de experiências de aprendizagem para o planejamento docente e aprendizagem discente: limites e desafios à prática educativa}

$\mathrm{Na}$ figura 1, observando o sistema em conjunto, percebemos as inter-relações entre as cinco etapas. Nessa situação, é possível perceber que o modelo Addie se apresenta como importante ferramenta no planejamento pedagógico. As etapas são dependentes entre si, pois cada uma alimenta a seguinte podendo comprometer o processo de aprendizagem, caso a etapa anterior não seja finalizada corretamente. Essa abordagem sistêmica garante um alinhamento das estratégias para que os objetivos sejam alcançados.

A primeira fase - análise - considerada a mais importante, é na qual todas as necessidades do discente deve ser compreendidas. Nela o discente está como foco principal. Quando se pode observar seu conteúdo, expectativas, necessidades, desejos e contexto social. Estipula-se os objetivos que depois de claramente destacados segue nova análise para se definir como alcança-los. O que se deve fazer para que cada grupo 
ou discente alcance o objetivo estipulado e como proporcionar uma melhor forma de aprendizagem. Nesta fase deve-se distinguir o que o discente já sabe e o que deverá saber ao completar o curso, disciplina ou período. Esta fase analise vários fatores que orientam o projeto de desenvolvimento do sistema de aprendizagem.

A fase seguinte - Design - consiste na seleção das ferramentas e recursos necessários para o alcance do desempenho discente, ou seja, inclui toda a abordagem pedagógica da ação educacional, abrangendo elementos como, a elaboração das atividades, conteúdos, a seleção das estratégias, artefatos, metodologias e técnicas que serão utilizadas no decorrer da ação.

Conforme Gomes e Silva (2016) as técnicas, tais como por exemplo, diário de autoobservação, questionários, entrevistas, observações e avaliação de desempenho, "são usadas para se capturar os pontos de vista de professores, alunos, coordenadores, diretores ou pais sobre algo relativo a aprendizagem, desenvolvimento de habilidades e competências, satisfação e emoção", assim, a utilização das técnicas resulta "em dados mais ou menos ricos sobre experiências de ensino-aprendizagem" (p. 130).

A fase posterior - Desenvolvimento - compreende a etapa de produção e elaboração dos objetos de aprendizagem. Nesta fase de elaboração e desenvolvimento concerne dois tipos segundo a amplitude: primeira fase simples que se desenvolvem as técnicas e ferramentas habituais do formador e na qual está contida a preparação e sua revisão. $\mathrm{Na}$ concepção de um projeto, esta será uma fase complexa pois envolve a seleção de conteúdo, o cenário das atividades pedagógicas, a fabricação dos recursos e o controle do uso dos recursos.

$\mathrm{Na}$ fase Implementação ocorre a operacionalização da ação educacional planejada, isto é, a aplicação da proposta educacional junto ao público-alvo. Nessa etapa, conforme Filatro (2008, p. 31) "envolve uma carga maior de metacognição (pensar sobre os próprios processos de aprendizagem) para tomada de decisões individuais ou colaborativas relacionadas ao design".

Assim sendo, a avaliação de uma situação de aprendizagem, sendo esta, diagnostica, formativa ou somativa deve permitir o aprimoramento de forma contínua das experiências de aprendizagem e, além do mais, colaborar em novas práticas de planejamento didático.

\section{Considerações finais}

O tipo de pesquisa metodológica usada nesse estudo foi predominantemente qualitativa e o procedimento utilizado foi bibliográfico, pois tínhamos interesse de descrever o modelo Addie e avaliar suas contribuições para prática docente, fazendo uso do design de experiência de aprendizagem no ensino a distância educação superior. 0 levantamento bibliográfico feito identificou que o modelo permitiu pôr em interação a pedagogia, a didática e a tecnologia propiciando ao discente um ambiente que proporciona o desenvolvimento de suas competências.

Conclui-se que o objetivo principal do presente estudo, ou seja, descrever o modelo Addie e avaliar seu uso no design de experiência de aprendizagem, foi alcançado, tendo em vista, que as contribuições para a educação superior são evidenciadas por meio da aprendizagem do discente e do melhor planejamento docente, pois as etapas se relacionam e são dependentes. Sendo assim, para prosseguir rumo ao alcance do

\begin{tabular}{|l|l|l|l|l|r|} 
Regae: Rev. Gest. Aval. Educ. & Santa Maria & v. 8 & n. 17 & Pub. contínua 2019 & p. 1-9
\end{tabular}


objetivo pedagógico mensurado na avaliação, o docente precisa cumprir as etapas anteriores, as quais as necessidades discentes são levantadas e levadas em consideração na etapa de design e de desenvolvimento. Para dar continuidade ao estudo, pretende-se aplicar uma entrevista semiestruturada a uma amostra de docentes que atua no ensino a distância para identificar suas percepções sobre o modelo Addie.

\section{Referências}

BERTALANFFY, Karl Ludwig von. Teoria geral dos sistemas. Petrópolis: Vozes, 2008.

BONFIM, David. Pedagogia no treinamento. Rio de Janeiro: Qualitymark, 1998.

FILATRO, Andrea. Design instrucional na prática. São Paulo: Pearson Education do Brasil, 2008.

GOMES, Alex Sandro; SILVA, Paulo André. Design de experiências de aprendizagem: criatividade e inovação para o planejamento das aulas. Recife: Pipa Comunicação, 2016.

LEBRUN, Marcel. Quality towards an expected harmony: pedagogy and technology speaking together about innovation. AACE Journal, 15(2), 115-130. Chesapeake, VA: Association for the Advancement of Computing in Education (AACE). Retrieved August 21, 2017. Disponível em https://www.learntechlib.org/p/21024/. Acesso em 10 ago. 2017.

LIBÂNEO, José Carlos. Democratização da escola pública: a pedagogia crítico-social dos conteúdos. São Paulo: Loyola, 1990.

LIMA, Marcos Antonio Martins. Auto-avaliação e desenvolvimento institucional na educação superior: projeto aplicado em cursos de administração. Fortaleza: UFC, 2008.

LUCKESI, Carlos Cipriano. Avaliação da aprendizagem escolar: estudos e proposições. São Paulo: Cortez, 2011.

FREIRE, Paulo. Pedagogia do oprimido. Rio de Janeiro: Paz e Terra, 2005.

KRAEMER, Maria Elisabeth Pereira. A avaliação da aprendizagem como processo construtivo de um novo fazer. Avaliação: Revista da Avaliação da Educação Superior, v. 10, n. 2, 2005, p. 137-147.

RAABE, André Luis Alice; GOMES, Alex Sandro; BITTENCOURT, Ig lbert; PONTUAL, Taciana. Educação criativa: multiplicando experiências para a aprendizagem. Recife: Pipa Comunicação, 2016.

SIMÃO NETO, Antonio; HESKETH, Camile Gonçalves. Didática e design instrucional. Curitiba: lesde, 2009.

SOUZA, Andréa Moura da Costa. Avaliação docente em estagio probatório: estudo das ações educacionais do programa CASA/UFC-comunidade de cooperação e aprendizagem significativa. Fortaleza: UFCE, 2016. 173f. Tese (doutorado em Educação). Universidade Federal do Ceará.

STUFFLEBEAM, Daniel. The CIPP model for evaluation: an update, a review of the model's development, a checklist to guide implementation. Paper read at Oregon Program Evaluators Network Conference, at Portland, Oregon, 2003. 
TYLER, Ralph. Princípios básicos do currículo e do ensino. Porto Alegre: Globo, 1974.

VIÉVILLE, Claude; PETER, Yves-Alain. Learning activity modeling and management. International Conference on Computers in Education (ICCE'02). IEEE. Auckland, New Zealand, 2002.

Andréa Moura da Costa Souza é professora e pesquisadora no Instituto Federal do Ceará, doutora em Educação Brasileira pela Universidade Federal do Ceará. Tem mestrado em Administração de Empresas e em Educação pela Université de Nancy 2, France.

Orcid: https://orcid.org/0000-0003-1059-0756.

Endereço: Rua Padre Roma 1055/401A - 60040-360 - Fortaleza - CE - Brasil.

E-mail: andrea.souza@ifce.edu.br.

Maria Lucijane Gomes de Oliveira tem mestrado em Educação Brasileira, especialização Gestão Escolar pela Universidade Estadual do Ceará e especialização em Design Educacional pelo Instituto Brasileiro de Desenho Instrucional.

Orcid: https://orcid.org/0000-0002-7136-7462.

Endereço: Alameda Jaime Augusto, 28 - 60332-180 - Fortaleza - CE - Brasil.

E-mail: luciijanne.oliveira@gmail.com.

Marcos Antonio Martins Lima é professor associado na Universidade Federal do Ceará, economista, mestre em Administração, doutor em Educação e pósdoutorado em Gestão. Coordenador do Grupo de Pesquisa em Avaliação \& Gestão Educacional vinculado aos Programas de Pós-Graduação em Educação e Administração e Controladoria da Universidade Federal do Ceará.

Orcid: https://orcid.org/0000-0001-5541-6220.

Endereço: Rua Dr. Gilberto Studart, 1290/902 - 60192-095 - Fortaleza - CE - Brasil. E-mail: marcos.a.lima@terra.com.br.

Blússia Tétis Brito Batista é graduada em Administração e colaboradora Gpage da Universidade Federal do Ceará.

Orcid: https://orcid.org/0000-0002-9239-833X.

Endereço: Rua Moreira de Sousa, 525/302 - 60450-080 - Fortaleza - CE - Brasil.

E-mail: blussia@hotmail.com.

Recebido em 30 de março de 2018.

Aceito em 15 de setembro de 2018.

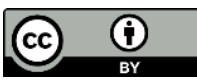

